\title{
BMJ Open Left atrial pressure overload and prognosis in elderly patients with heart failure and preserved ejection fraction: a prospective multicenter observational study
}

Shiro Hoshida (D) , ${ }^{1}$ Koichi Tachibana, ${ }^{1}$ Yukinori Shinoda, ${ }^{1}$ Tomoko Minamisaka, ${ }^{1}$ Takahisa Yamada, ${ }^{2}$ Yoshiharu Higuchi, ${ }^{3}$ Yusuke Nakagawa, ${ }^{4}$ Haruhiko Abe, ${ }^{5}$ Hisakazu Fuji, ${ }^{6}$ Yoshio Yasumura, ${ }^{7}$ Shungo Hikoso (D) , ${ }^{8}$ Daisaku Nakatani, ${ }^{8}$ Yasushi Sakata (D), ${ }^{8}$ Osaka Cardiovascular Conference Investigators, On behalf of the Osaka Cardiovascular Conference Investigators

To cite: Hoshida S, Tachibana K, Shinoda Y, et al. Left atrial pressure overload and prognosis in elderly patients with heart failure and preserved ejection fraction: a prospective multicenter observational study. BMJ Open 2021;11:e044605. doi:10.1136/ bmjopen-2020-044605

- Prepublication history for this paper is available online. To view these files, please visit the journal online (http://dx.doi. org/10.1136/bmjopen-2020044605).

Received 10 September 2020 Accepted 11 September 2021

A) Check for updates

(c) Author(s) (or their employer(s)) 2021. Re-use permitted under CC BY-NC. No commercial re-use. See rights and permissions. Published by BMJ.

For numbered affiliations see end of article.

Correspondence to Dr Shiro Hoshida; shiro.hoshida@hosp-yao. osaka.jp

\section{ABSTRACT}

Objectives The severity of diastolic dysfunction is assessed using a combination of several indices of left atrial (LA) volume overload and LA pressure overload. We aimed to clarify which overload is more associated with the prognosis in patients with heart failure and preserved ejection fraction (HFpEF).

Setting A prospective, multicenter observational registry of collaborating hospitals in Osaka, Japan.

Participants We enrolled hospitalised patients with HFpEF showing sinus rhythm (men, 79; women, 113). Blood tests and transthoracic echocardiography were performed before discharge. The ratio of diastolic elastance $(\mathrm{Ed})$ to arterial elastance (Ea) was used as a relative index of LA pressure overload.

Primary outcome measures All-cause mortality and admission for heart failure were evaluated at $>1$ year after discharge.

Results In the multivariable Cox regression analysis, Ed/ $\mathrm{Ea}$, but not LA volume index, was significantly associated with all-cause mortality or admission for heart failure (HR $2.034,95 \% \mathrm{Cl} 1.059$ to $3.907, \mathrm{p}=0.032$ ), independent of age, sex, and the serum $\mathrm{N}$-terminal pro-brain natriuretic peptide (NT-proBNP) level. In patients with a higher NTproBNP level, the effect of higher Ed/Ea on prognosis was prominent $(p=0.015)$.

Conclusions Ed/Ea, an index of LA pressure overload, was significantly associated with the prognosis in elderly patients with HFpEF showing sinus rhythm.

Trial registration number UMIN000021831.

\section{INTRODUCTION}

Patients with heart failure and preserved ejection fraction (HFpEF) have an increased left atrial volume (LAV) and early transmitral flow velocity/the onset of early diastolic mitral annular velocity (E/é), as shown by noninvasive echocardiographic findings. ${ }^{1-3} \mathrm{E} / \mathrm{e}$ is positively correlated with left atrial (LA)
Strengths and limitations of this study

- The severity of diastolic dysfunction is assessed by a combination of several indices of left atrial (LA) volume and pressure overload.

- The ratio of diastolic elastance (Ed) to arterial elastance (Ea), that is, Ed/Ea, is a novel index of LA pressure overload.

- Although the indices of LA pressure and volume overload are high in patients with heart failure and preserved ejection fraction (HFpEF), it remains to be seen which LA overload is more associated with the prognosis in elderly patients with $\mathrm{HFpEF}$.

- The limitation of this study is its small sample size.

pressure or pulmonary capillary wedge pressure. ${ }^{4-7}$ We previously reported that the LAV index (LAVI), a relative index of LAV overload, and the ratio of diastolic elastance (Ed) to arterial elastance (Ea) $(\mathrm{Ed} / \mathrm{Ea}=(\mathrm{E} / \mathrm{e}) /$ $(0.9 \times$ systolic blood pressure $))$, a relative index of LA pressure overload, are high in elderly patients with preserved ejection fraction with and without heart failure (HF). ${ }^{389}$ In the recommendations for left ventricular (LV) diastolic evaluation using echocardiography, the severity of diastolic dysfunction (DD) is assessed using a combination of several indices, such as early transmitral flow (E)/late transmitral flow (A), deceleration time, E/é, tricuspid regurgitation velocity and LAVI. ${ }^{7}{ }^{10}$ Evaluation of disease severity based on these recommendations is useful for estimating the prognosis of patients with HFpEF. ${ }^{11}$ However, these non-invasive indices are related to either LA pressure overload or LAV overload, and which overload is more associated with 
the prognosis of these patients remains unclear. In this study, we aimed to identify a clinically significant echocardiographic index of LA pressure or volume overload for the prognosis of patients with HFpEF.

\section{METHODS}

\section{Study subjects}

Of the 353 patients with prognostic data who were recruited from the Prospective Multicenter Observational Study of Patients with Heart Failure and Preserved Ejection Fraction (PURSUIT HFpEF) registry, ${ }^{3}{ }^{12} 129$ patients were excluded because they showed atrial fibrillation before discharge and 32 patients were excluded because of poor echocardiographic data. Therefore, we enrolled 192 patients showing sinus rhythm (LV ejection fraction (LVEF) $\geq 50 \%$; men/women, 79/113; mean age, 80 years) at discharge during the index hospitalisation with acute decompensated $\mathrm{HF}$; patients were enrolled based on the Framingham criteria, and if they met the criteria of LVEF $\geq 50 \%$ on transthoracic echocardiography (TTE) and N-terminal pro-brain natriuretic peptide (NT-proBNP) $\geq 400 \mathrm{pg} / \mathrm{mL}$ on admission. We excluded patients with severe aortic stenosis, aortic regurgitation, mitral stenosis or mitral regurgitation due to structural changes in the valves detected by TTE on admission. The PURSUIT HFpEF registry is a prospective, multicenter observational registry in which collaborating hospitals in Osaka, Japan recorded clinical, echocardiographic and outcome data of patients with HFpEF. The registry was managed in accordance with the Declaration of Helsinki.

\section{Echocardiography and laboratory testing}

TTE was performed when the patients were in a stable condition before discharge. Echocardiographic measurements were obtained according to the American Society of Echocardiography or European Society of Echocardiography criteria during a stable sinus rhythm. ${ }^{10}{ }^{13}$ Volumetry was standardised using the modified Simpson's method, and the index was calculated as the LAV divided by the body surface area. As a marker of LA pressure overload for estimating LV diastolic function, we examined afterload-integrated $\mathrm{Ed} / \mathrm{Ea}$ ((E/é)/(0.9×systolic blood pressure) ). ${ }^{3914}$ As relative markers of LAV overload, we also evaluated LAVI and LA ejection fraction calculated as stroke volume $(\mathrm{SV}) / \mathrm{LAV}^{15}$ The severity of LVDD was assessed according to the previous reports. ${ }^{10} 11$ In the first step, the following four parameters were used: E/é, é velocity, tricuspid regurgitation velocity and LAVI. In the second step, E/A, E wave, E/é, tricuspid regurgitation velocity and LAVI were used to determine DD grades $1-3 .{ }^{10} 11$ When DD was not observed in the first step, the patients were classified as DD grade 0. Laboratory data were examined when patients were stable before discharge.

\section{Follow-up/clinical outcome}

After discharge, all patients were followed up at the respective hospital. Survival data were obtained by dedicated coordinators and investigators through direct contact with patients or their physicians at the hospital, or in an outpatient setting, or via a telephone interview with their families or by mail. Data collection was performed using an electronic data capture system integrated into the electronic medical records developed at the Osaka University. ${ }^{16}$ In-hospital data were entered into the system and transferred to the data collection centre via a secure Internet connection for processing and analysis. The primary endpoints of this study were the composite of allcause mortality and hospitalisation for worsening HF.

\section{Patient and public involvement \\ No patient involved.}

\section{Statistical analysis}

Continuous variables are expressed as mean $\pm \mathrm{SD}$, whereas categorical variables are presented as frequencies and percentages. Differences in categorical variables between the groups were assessed using the $\chi^{2}$ test, and those in continuous variables were assessed using Student's t-test or Welch's t-test, as appropriate. Coefficients of correlations were assessed using the Pearson or Spearman model, and $p$ values were examined using regression analysis. Survival curves were estimated using the Kaplan-Meier productlimit estimator, and the groups were compared using the log-rank test. The Cox HR was evaluated using univariable and multivariable analyses. In the multivariable analysis, age and sex, and NT-proBNP level, LAVI and Ed/Ea that were significantly associated with outcome in the univariable analysis were included. A p value of $<0.05$ was considered statistically significant. All statistical analyses were performed using EZR (Saitama Medical Centre, Jichi Medical University, Saitama, Japan), which is a graphical user interface for $\mathrm{R}$ (The $\mathrm{R}$ Foundation for Statistical Computing, Vienna, Austria).

\section{RESULTS}

\section{Clinical and laboratory characteristics of patients with HFpEF}

During a median follow-up of 452 days, 50 patients had all-cause mortality or admission for worsening HF. There were significant differences between patients with and without all-cause mortality or admission for HF in terms of age $(\mathrm{p}=0.011)$, estimated glomerular filtration rate (eGFR) $(\mathrm{p}=0.026)$ and serum NT-proBNP $(\mathrm{p}=0.017)$ and albumin $(\mathrm{p}<0.001)$ levels (table 1$)$. There were no significant differences in medications or the incidence of hypertension and dyslipidaemia, except for diabetes mellitus, between the two groups. With respect to echocardiographic parameters, LAVI $(\mathrm{p}=0.024)$, tricuspid regurgitation pressure gradient (TRPG, $\mathrm{p}<0.001$ ) and $\mathrm{Ed} / \mathrm{Ea}$ ( $p=0.019$ ) but not SV/LAV, LV mass index (LVMI), LVEF, $\mathrm{E} / \mathrm{A}$, the deceleration time of the $\mathrm{E}$ wave, septal $\mathrm{e}^{\prime}$, lateral $\mathrm{e}^{\prime}$, or $\mathrm{Ed}=\left(\mathrm{E} / \mathrm{e}^{\prime}\right) / \mathrm{SV}$ at discharge, were significantly 
Table 1 Patient characteristics before discharge

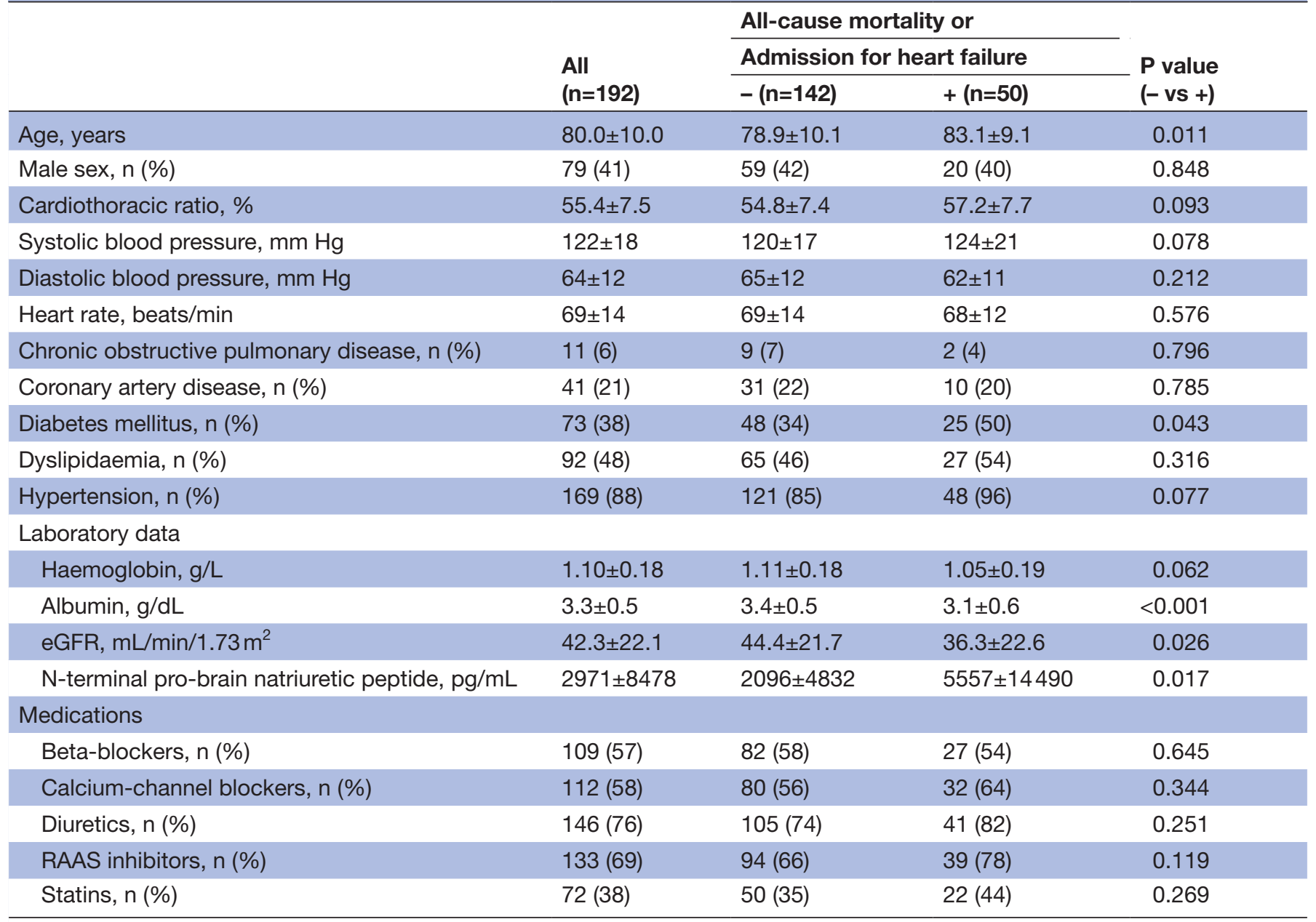

Values are mean \pm SD or number (\%).

eGFR, estimated glomerular filtration rate; RAAS, renin-angiotensin-aldosterone system.

different between patients with and without all-cause mortality or admission for HF (table 2).

The correlations between Ed/Ea and LAVI ( $r=0.194$, $\mathrm{p}=0.008)$ or $\mathrm{SV} / \mathrm{LAV}(\mathrm{r}=-0.180, \mathrm{p}=0.017)$ were more significant than those between $\mathrm{E} / \mathrm{e}^{\prime}$ and LAVI $(\mathrm{r}=0.155$, $\mathrm{p}=0.034)$ or $\mathrm{SV} / \mathrm{LAV}(\mathrm{r}=-0.137, \mathrm{p}=0.072) . \mathrm{E} / \mathrm{e}^{\prime}(\mathrm{r}=0.233$, $\mathrm{p}=0.001)$ and $\mathrm{Ed} / \mathrm{Ea}(\mathrm{r}=0.222, \mathrm{p}=0.002)$ showed a modest positive correlation with the NT-proBNP logtransformed level, although TRPG did not correlate with the NT-proBNP log-transformed level ( $\mathrm{r}=0.147, \mathrm{p}=0.060)$. LAVI and the NT-proBNP log-transformed level were significantly correlated $(\mathrm{r}=0.256, \mathrm{p}<0.001)$.

\section{Prognostic analysis}

In the receiver operating characteristic (ROC) curve analysis for the prediction of all-cause mortality or admission for HF, the area under the curve of LAVI was slightly smaller than that of the NT-proBNP level and Ed/ Ea (table 3). The Kaplan-Meier survival analysis clearly showed that LAVI $>38 \mathrm{~mL} / \mathrm{m}^{2} \quad(\mathrm{p}=0.016), \mathrm{Ed} / \mathrm{Ea}>0.121$ $(\mathrm{p}=0.002)$ and NT-proBNP level $>783 \mathrm{pg} / \mathrm{mL}(\mathrm{p}<0.001)$ were significant for prognosis (figure 1). Although not shown, age $>85$ years $(\mathrm{p}<0.001)$, eGFR $<39.8 \mathrm{~mL} /$ $\min / 1.73 \mathrm{~m}^{2}(\mathrm{p}=0.004)$ and TRPG $>28 \mathrm{~mm} \mathrm{Hg}(\mathrm{p}<0.001)$ were also determinant factors. The albumin level was not a determinant factor (data not shown). The LVDD grade was also related to all-cause mortality or admission for HF in patients with HFpEF, as shown by the Kaplan-Meier survival curve analysis (figure 1) and Cox hazard analysis (HR 3.164, 95\% CI 1.761 to 5.683 , $\mathrm{p}<0.001)$. In the multivariable analysis of the Cox HR, $\mathrm{Ed} / \mathrm{Ea}(\mathrm{p}=0.032)$ was significantly associated with poor outcome, independent of age, sex, LAVI and serum NT-proBNP level (table 3).

In the Kaplan-Meier survival curve analysis for all-cause mortality or admission for HF with a stratified examination using the NT-proBNP level and Ed/Ea, the patients with NT-proBNP level $>783 \mathrm{pg} / \mathrm{mL}$ and $\mathrm{Ed} / \mathrm{Ea}>0.121$ exhibited the highest event rate (figure 2, log-rank test, $\mathrm{p}=0.015)$. The effect of higher $\mathrm{Ed} / \mathrm{Ea}$ on all-cause mortality or admission for HF was obvious in patients with a higher NT-proBNP level. 
Table 2 Echocardiographic data before discharge

\begin{tabular}{|c|c|c|c|c|}
\hline & \multirow[b]{3}{*}{ All } & \multicolumn{2}{|c|}{ All-cause mortality or } & \multirow{3}{*}{$\begin{array}{l}\text { P value } \\
\text { (- vs }+)\end{array}$} \\
\hline & & \multicolumn{2}{|c|}{ Admission for heart failure } & \\
\hline & & - & + & \\
\hline LAD, mm & $41.2 \pm 7.6$ & $40.4 \pm 7.9$ & $43.3 \pm 6.5$ & 0.021 \\
\hline LAVI, mL/m² & $50.5 \pm 25.7$ & $47.9 \pm 23.2$ & $57.6 \pm 30.8$ & 0.024 \\
\hline LVEDVI, $\mathrm{mL} / \mathrm{m}^{2}$ & $56.1 \pm 20.3$ & $55.9 \pm 21.2$ & $56.8 \pm 17.6$ & 0.786 \\
\hline LVESVI, mL/m² & $21.8 \pm 10.8$ & $21.8 \pm 10.9$ & $21.8 \pm 10.7$ & 0.993 \\
\hline $\mathrm{SVI}, \mathrm{mL} / \mathrm{m}^{2}$ & $34.3 \pm 12.0$ & $34.0 \pm 12.7$ & $35.0 \pm 10.0$ & 0.652 \\
\hline SV/LAV & $0.809 \pm 0.376$ & $0.835 \pm 0.376$ & $0.733 \pm 0.373$ & 0.125 \\
\hline LVEF, \% & $61.4 \pm 6.8$ & $61.3 \pm 6.7$ & $62.0 \pm 6.8$ & 0.502 \\
\hline LVMI, $g / \mathrm{m}^{2}$ & $108.4 \pm 33.2$ & $105.8 \pm 32.5$ & $115.9 \pm 34.1$ & 0.063 \\
\hline TRPG, $\mathrm{mm} \mathrm{Hg}$ & $27.2 \pm 9.3$ & $25.8 \pm 8.5$ & $30.9 \pm 10.4$ & $<0.001$ \\
\hline $\mathrm{E} / \mathrm{A}$ & $1.00 \pm 0.57$ & $1.00 \pm 0.61$ & $1.01 \pm 0.47$ & 0.897 \\
\hline DcT of E wave & $0.22 \pm 0.06$ & $0.22 \pm 0.06$ & $0.22 \pm 0.07$ & 0.468 \\
\hline Septal $e^{\prime}$ & $0.051 \pm 0.019$ & $0.052 \pm 0.020$ & $0.048 \pm 0.016$ & 0.189 \\
\hline Lateral $e^{\prime}$ & $0.067 \pm 0.023$ & $0.067 \pm 0.024$ & $0.067 \pm 0.020$ & 0.979 \\
\hline$E d=\left(E / e^{\prime}\right) / S V$ & $0.450 \pm 0.230$ & $0.431 \pm 0.227$ & $0.505 \pm 0.249$ & 0.065 \\
\hline $\mathrm{Ed} / \mathrm{Ea}$ & $0.130 \pm 0.055$ & $0.125 \pm 0.055$ & $0.146 \pm 0.052$ & 0.019 \\
\hline
\end{tabular}

Values are mean \pm SD.

DcT, deceleration time; E, early transmitral flow velocity; e', onset of early diastolic mitral annular velocity; Ea, arterial elastance; Ed, diastolic elastance; LAD, left atrial diameter; LAV, left atrial volume; LAVI, left atrial volume index; LVEDVI, left ventricular end-diastolic volume index; LVEF, left ventricular ejection fraction; LVESVI, left ventricular end-systolic volume index; SV, stroke volume; SVI, stroke volume index; TRPG, tricuspid regurgitation pressure gradient.

\section{DISCUSSION}

In this study, LA pressure overload, rather than LAV overload, was found to be a more useful marker of prognosis in patients with HFpEF. Our findings can help determine which single index of LA pressure overload is significantly associated with the prognosis. In particular, in patients with a higher NT-proBNP level, a higher Ed/Ea was associated with a poor prognosis.

The heterogeneity of the cardiac structure in patients with HFpEF is well known. Notably, there were no significant differences in the deceleration time of the $\mathrm{E}$ wave and $\mathrm{E} / \mathrm{A}$ in patients with and without all-cause mortality or admission for HF. The LA structure and function most closely reflect haemodynamic stress and remodelling in HFpEF. ${ }^{17}$ The $\mathrm{E} / \mathrm{e}^{\prime}$ ratio was reported to be a significant prognostic factor in the Treatment of Preserved Cardiac Function Heart Failure with an Aldosterone Antagonist (TOPCAT) trial ${ }^{18}$ and a systematic review. ${ }^{19}$ However, there are many important differences between our study and the TOPCAT trial: (1) the TOPCAT trial was an intervention study; (2) subjects in our study were 10 years older; (3) the inclusion criteria were different (ie, stable outpatients in the TOPCAT trial vs hospitalised patients with HFpEF in our study and patients with

Table 3 Analytical data of prognostic factors for all-cause mortality or admission for heart failure in patients with heart failure and preserved ejection fraction

\begin{tabular}{|c|c|c|c|c|c|c|c|c|}
\hline & & & \multicolumn{6}{|c|}{ Cox hazard analysis } \\
\hline & \multicolumn{2}{|c|}{ ROC curve analysis } & \multicolumn{3}{|c|}{ Univariable } & \multicolumn{3}{|c|}{ Multivariable } \\
\hline & Cut-off point & AUC & Ratio & $95 \% \mathrm{Cl}$ & $P$ value & Ratio & $95 \% \mathrm{Cl}$ & $P$ value \\
\hline Age & 85 & 0.628 & 2.855 & 1.634 to 4.99 & $<0.001$ & 1.736 & 0.934 to 3.225 & 0.081 \\
\hline Sex & - & - & 0.965 & 0.547 to 1.701 & 0.903 & 1.223 & 0.638 to 2.345 & 0.544 \\
\hline NT-proBNP & 783 & 0.695 & 3.432 & 1.652 to 7.133 & $<0.001$ & 3.152 & 1.422 to 6.987 & 0.004 \\
\hline LAVI & 38 & 0.607 & 2.225 & 1.134 to 4.366 & 0.02 & 1.298 & 0.599 to 2.813 & 0.508 \\
\hline $\mathrm{Ed} / \mathrm{Ea}$ & 0.121 & 0.637 & 2.424 & 1.337 to 4.394 & 0.003 & 2.034 & 1.059 to 3.907 & 0.032 \\
\hline
\end{tabular}

AUC, area under the curve; Ea, arterial elastance; Ed, diastolic elastance; LAVI, left atrial volume index; NT-proBNP, N-terminal pro-brain natriuretic peptide; ROC, receiver operating characteristic. 

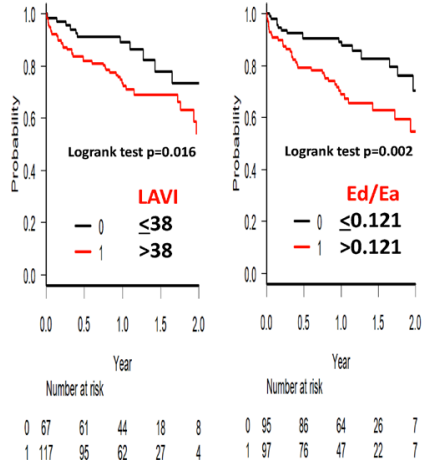

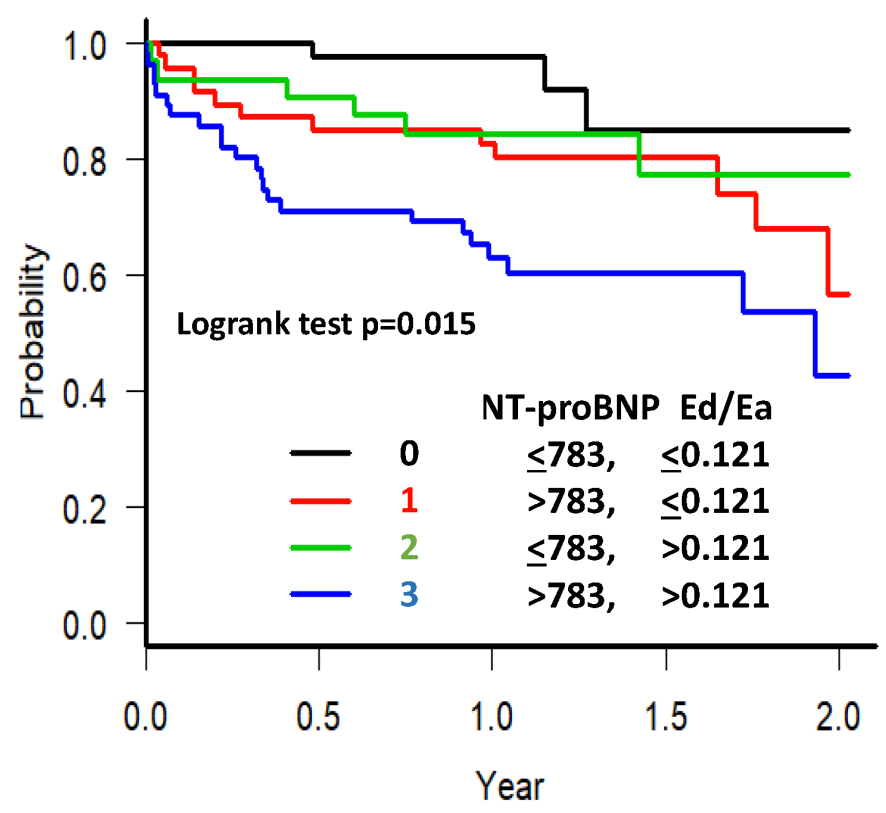

Number at risk

$\begin{array}{llllll}\mathbf{0} & 43 & 42 & 30 & 11 & 4 \\ 1 & 47 & 40 & 32 & 13 & 3 \\ 2 & 32 & 29 & 19 & 11 & 4 \\ 3 & 56 & 39 & 25 & 10 & 2\end{array}$

Figure 2 The Kaplan-Meier survival curve analysis for allcause mortality or admission for heart failure with stratified examination using the ratio of Ed/Ea and NT-proBNP level in patients with heart failure and preserved ejection fraction. Patients with NT-proBNP level $>783 \mathrm{pg} / \mathrm{mL}$ and Ed/Ea $>0.121$ exhibited higher all-cause mortality or admission for heart failure. In patients with a higher NT-proBNP level, the effect of a higher $\mathrm{Ed} / \mathrm{Ea}$ on all-cause mortality or admission for heart failure was obvious. Ea, arterial elastance; Ed, diastolic elastance; NT-proBNP, N-terminal pro-brain natriuretic peptide.

multivariable analysis of the Cox HR for all-cause mortality or admission for $\mathrm{HF}$ in patients with $\mathrm{HFpEF}$, we conclude that an index of LA volume overload such as LAVI is not a suitable factor for evaluating prognosis. LAVI is an indicator of long-term elevation of LV filling pressure, and an enlarged LAVI may be a secondary phenomenon. Even in patients without all-cause mortality or admission for $\mathrm{HF}$, the mean LAVI was $47.9 \mathrm{~mL} / \mathrm{m}^{2}$, which was considerably higher than the criterion for LVDD $\left(>34 \mathrm{~mL} / \mathrm{m}^{2}\right)$.

$\mathrm{LV} \mathrm{Ed}$ is expressed as $\left(\mathrm{E} / \mathrm{e}^{\prime}\right) / \mathrm{SV}^{25}$ or $\left(\mathrm{E} / \mathrm{e}^{\prime}\right) / \mathrm{LV}$ enddiastolic volume. ${ }^{26}$ Ea was calculated as $(0.9 \times$ systolic blood pressure) $/ \mathrm{SV}^{25}$ Although Ed and Ea were reported to be negatively correlated in younger patients with hypertension, ${ }^{27}$ both indices were higher in elderly women than in men under stable conditions. ${ }^{25} 26$ Elevated Ed in elderly women could be an epiphenomenon because of the associated increase in Ea. We previously reported that Ed/ $\mathrm{Ea}$ is an index of the $\mathrm{LV}$ diastolic function relative to the afterload and can be calculated as $(\mathrm{E} / \mathrm{ev}) /(0.9 \times$ systolic blood pressure) when $\mathrm{Ed}$ is $\left(\mathrm{E} / \mathrm{e}^{\prime}\right) / \mathrm{SV}^{8}{ }^{9}$ Accordingly, $\mathrm{Ed} / \mathrm{Ea}$ was not directly related to the parameters of 
cardiac volume, such as LAV and SV. We recently reported a larger LAV and higher Ed/Ea in elderly women with preserved ejection fraction, regardless of HF status. ${ }^{3} 89$ $\mathrm{Ed} / \mathrm{Ea}$ is a novel afterload-integrated parameter for $\mathrm{LV}$ diastolic function that may be useful as a severity index for prognosis in elderly patients with HFpEF.

\section{Limitations}

Further studies are required to investigate differences in the clinical significance of $\mathrm{Ed} / \mathrm{Ea}$ for prognosis between younger patients with normal renal function and moderate-to-severe LV hypertrophy and elderly patients (mean age, 80 years) with renal dysfunction (mean eGFR, $42.3 \mathrm{~mL} / \mathrm{min} / 1.73 \mathrm{~m}^{2}$ ) and mild LV hypertrophy (mean LVMI, $108.4 \mathrm{~g} / \mathrm{m}^{2}$ ) included in our study. We could not discuss echocardiographic parameters in patients with atrial fibrillation. The role of the right side of the heart in prognosis, as possibly reflected in the involvement of TRPG, remains unclear in this study. Even in the small sample size, the multivariable Cox model with the number of variables included/input was within the rough rule of one variable per 10 events. Under this condition, $\mathrm{Ed} /$ Ea was a significant prognostic factor, independent of NT-proBNP level. Although our results need to be interpreted carefully, our finding that a higher $\mathrm{Ed} / \mathrm{Ea}$ was associated with a poor prognosis in patients with a higher NT-proBNP level may be clinically important. We examined all-cause mortality rather than cardiac death because the determination of cardiac death can be challenging in elderly patients.

\section{CONCLUSIONS}

LA pressure overload, rather than LAV overload, is a useful marker of prognosis in elderly patients with $\mathrm{HFpEF}$ showing sinus rhythm. As an index for LA pressure overload among non-invasive echocardiographic findings, $\mathrm{Ed} /$ Ea provides additional prognostic information on the serum NT-proBNP level.

\section{Author affiliations}

${ }^{1}$ Department of Cardiovascular Medicine, Yao Municipal Hospital, Yao, Osaka, Japan ${ }^{2}$ Division of Cardiology, Osaka General Medical Center, Osaka, Osaka, Japan ${ }^{3}$ Cardiovascular Division, Osaka Police Hospital, Osaka, Osaka, Japan ${ }^{4}$ Division of Cardiology, Kawanishi City Hospital, Kawanishi, Hyogo, Japan ${ }^{5}$ Department of Cardiovascular Medicine, National Hospital Organization Osaka National Hospital, Osaka, Osaka, Japan

${ }^{6}$ Cardiovascular Division, Kobe Ekisaikai Hospital, Kobe, Hyogo, Japan ${ }^{7}$ Division of Cardiology, Amagasaki Chuo Hospital, Amagasaki, Hyogo, Japan ${ }^{8}$ Department of Cardiovascular Medicine, Osaka University Graduate School of Medicine, Suita, Osaka, Japan

Acknowledgements We thank Hiroyuki Kurakami and Tomomi Yamada for their statistical support. We also thank Nagisa Yoshioka, Kyoko Tatsumi, Satomi Kishimoto, Noriko Murakami and Sugako Mitsuoka for their excellent assistance with data collection.

Collaborators Shunsuke Tamaki, Masatake Fukunami, and Takahisa Yamada, Osaka General Medical Center, Osaka, Japan; Takaharu Hayashi and Yoshiharu Higuchi, Osaka Police Hospital, Osaka, Japan; Masaharu Masuda, Mitsutoshi Asai, and Toshiaki Mano, Kansai Rosai Hospital, Amagasaki, Japan; Hisakazu Fuji, Kobe Ekisaikai Hospital, Kobe, Japan; Daisaku Masuda, Yoshihiro Takeda,
Yoshiyuki Nagai, and Shizuya Yamashita, Rinku General Medical Center, Izumisano, Japan; Masami Sairyo, Yusuke Nakagawa, and Shuichi Nozaki, Kawanishi City Hospital, Kawanishi, Japan; Haruhiko Abe, Yasunori Ueda, Masaaki Uematsu, and Yukihiro Koretsune, National Hospital Organization Osaka National Hospital, Osaka, Japan; Kunihiko Nagai, Ikeda Municipal Hospital, Ikeda, Japan; Masamichi Yano, Masami Nishino, and Jun Tanouchi, Osaka Rosai Hospital, Sakai, Japan; Yoh Arita and Shinji Hasegawa, Japan Community Health Care Organization Osaka Hospital, Osaka, Japan; Takamaru Ishizu, Minoru Ichikawa, and Yuzuru Takano Higashiosaka City Medical Center, Higashiosaka, Japan; Eisai Rin, Kawachi General Hospital, Higashiosaka, Japan; Tetsuya Watanabe and Shiro Hoshida, Yao Municipal Hospital, Yao, Japan; Masahiro Izumi, Kinki Central Hospital, Itami, Japan; Hiroyoshi Yamamoto and Hiroyasu Kato, Japan Community Health Care Organization, Osaka Minato Central Hospital, Osaka, Japan; Kazuhiro Nakatani and Hisatoyo Hiraoka, Sumitomo Hospital, Osaka, Japan; Mayu Nishio and Keiji Hirooka, Saiseikai Senri Hospital, Suita, Japan; Takahiro Yoshimura and Yoshinori Yasuoka, National Hospital Organization Osaka Minami Medical Center, Kawachinagano, Japan; Akihiro Tani, Kano General Hospital, Osaka, Japan; Yasushi Okumoto and Hideharu Akagi, Kinan Hospital, Tanabe, Japan; Yasunaka Makino, Hyogo Prefectural Nishinomiya Hospital, Nishinomiya, Japan; Toshinari Ohnishi and Katsuomi Iwakura, Sakurabashi Watanabe Hospital, Osaka, Japan; Nagahiro Nishikawa and Yoshiyuki Kijima, Japan Community Health Care Organization, Hoshigaoka Medical Center, Hirakata, Japan; Takashi Kitao and Hideyuki Kanai, Minoh City Hospital, Minoh, Japan; Wataru Shioyama and Masashi Fujita, Osaka International Cancer Institute, Osaka, Japan; Koichiro Harada, Suita Municipal Hospital, Suita, Japan; Masahiro Kumada and Osamu Nakagawa, Toyonaka Municipal Hospital, Toyonaka, Japan; Ryo Araki and Takayuki Yamada, Otemae Hospital, Osaka, Japan; Akito Nakagawa and Yoshio Yasumura, Amagasaki Chuo Hospital, Amagasaki, Japan; Fusako Sera, Kei Nakamoto, Hidetaka Kioka, Tomohito Ohtani, Katsuki Okada, Hiroya Mizuno, Tomoharu Dohi, Takayuki Kojima, Hirota Kida, Akihiro Sunaga, Oeun Bolrathanak, Shinichiro Suna, Daisaku Nakatani, Shungo Hikoso, Toshihiro Takeda, Yasushi Matsumura, and Yasushi Sakata, Osaka University Graduate School of Medicine, Suita, Japan.

Contributors (1) Conception and design of the study, acquisition of, and/or analysis and interpretation of data: SH, KT, YS, TM, YH, YN, HA and HF. (2) Discuss on the planning, drafting the article and/or revising it critically for important intellectual content: SH, TY, YY, SH, DN and YS. (3) Final approval of the version to be submitted: All authors.

Funding This study was funded by Roche Diagnostics KK and FUJIFILM Toyama Chemical. No grant numbers were applicable.

Competing interests None declared.

Patient and public involvement Patients and/or the public were not involved in the design, or conduct, or reporting, or dissemination plans of this research.

Patient consent for publication Not applicable.

Ethics approval This protocol (Osaka University Clinical Research Review Committee, R000024414) was approved by the ethics committee of Yao Municipal Hospital (2016-No.0006). All participants provided written informed consent.

Provenance and peer review Not commissioned; externally peer reviewed.

Data availability statement Data are available upon reasonable request. № additional data available.

Open access This is an open access article distributed in accordance with the Creative Commons Attribution Non Commercial (CC BY-NC 4.0) license, which permits others to distribute, remix, adapt, build upon this work non-commercially, and license their derivative works on different terms, provided the original work is properly cited, appropriate credit is given, any changes made indicated, and the use is non-commercial. See: http://creativecommons.org/licenses/by-nc/4.0/.

ORCID iDs

Shiro Hoshida http://orcid.org/0000-0002-0268-9417

Shungo Hikoso http://orcid.org/0000-0003-2284-1970

Yasushi Sakata http://orcid.org/0000-0002-5618-4721

\section{REFERENCES}

1 Nagueh SF, Middleton KJ, Kopelen HA, et al. Doppler tissue imaging: a noninvasive technique for evaluation of left ventricular relaxation and estimation of filling pressures. J Am Coll Cardiol 1997;30:1527-33. 
2 Geske JB, Sorajja P, Nishimura RA, et al. Evaluation of left ventricular filling pressures by Doppler echocardiography in patients with hypertrophic cardiomyopathy: correlation with direct left atria pressure measurement at cardiac catheterization. Circulation 2007;116:2702-8.

3 Hoshida S, Watanabe T, Shinoda Y, et al. Sex-Related differences in left ventricular diastolic function and arterial elastance during admission in patients with heart failure with preserved ejection fraction: the pursuit HFpEF study. Clin Cardiol 2018;41:1529-36.

4 Santos M, Rivero J, McCullough SD, et al. E/e' ratio in patients with unexplained dyspnea: lack of accuracy in estimating left ventricular filling pressure. Circ Heart Fail 2015;8:749-56.

5 Sharifov OF, Schiros CG, Aban I, et al. Diagnostic accuracy of tissue Doppler index $E / e^{\prime}$ for evaluating left ventricular filling pressure and diastolic Dysfunction/Heart failure with preserved ejection fraction: a systematic review and meta-analysis. J Am Heart Assoc 2016;5:e002530.

6 Obokata M, Kane GC, Reddy YNV, et al. Role of diastolic stress testing in the evaluation for heart failure with preserved ejection fraction: a simultaneous Invasive-Echocardiographic study. Circulation 2017;135:825-38.

7 Andersen OS, Smiseth OA, Dokainish $\mathrm{H}$, et al. Estimating Left Ventricular Filling Pressure by Echocardiography. J Am Coll Cardiol 2017;69:1937-48.

8 Hoshida S, Shinoda Y, Ikeoka K, et al. Age- and sex-related differences in diastolic function and cardiac dimensions in a hypertensive population. ESC Heart Fail 2016;3:270-7.

9 Hoshida S, Shinoda Y, Ikeoka K, et al. Fluctuation of Dynamic Diastolic Function Relative to Static Cardiac Structure - New Insights Into the Underlying Mechanism of Heart Failure With Preserved Ejection Fraction in Elderly Patients. Circ J 2017;81:755-8.

10 Nagueh SF, Smiseth OA, Appleton CP, et al. Recommendations for the Evaluation of Left Ventricular Diastolic Function by Echocardiography: An Update from the American Society of Echocardiography and the European Association of Cardiovascular Imaging. J Am Soc Echocardiogr 2016;29:277-314.

11 Sanchis L, Andrea R, Falces C, et al. Differential clinical implications of current recommendations for the evaluation of left ventricular diastolic function by echocardiography. J Am Soc Echocardiogr 2018;31:1203-8.

12 Suna S, Hikoso S, Yamada T, et al. Study protocol for the PURSUITHFpEF study: a prospective, multicenter, observational study of patients with heart failure with preserved ejection fraction. BMJ Open 2020;10:e038294.

13 Lang RM, Badano LP, Mor-Avi V, et al. Recommendations for cardiac chamber quantification by echocardiography in adults: an update from the American Society of echocardiography and the European association of cardiovascular imaging. J Am Soc Echocardiogr 2015;28:1-39.
14 Minamisaka T, Watanabe T, Shinoda Y, et al. Transient manifestation of left ventricular diastolic dysfunction following ablation in patients with paroxysmal atrial fibrillation. Clin Cardiol 2018;41:978-84.

15 Hoshida S, Watanabe T, Shinoda Y, et al. Considerable scatter in the relationship between left atrial volume and pressure in heart failure with preserved left ventricular ejection fraction. Sci Rep 2020;10:90.

16 Matsumura Y, Hattori A, Manabe S, et al. Case report form reporter: a key component for the integration of electronic medical records and the electronic data capture system. Stud Health Technol Inform 2017;245:516-20.

17 Abbasi SA, Shah RV, McNulty SE, et al. Left atrial structure and function in heart failure with preserved ejection fraction: a relax substudy. PLoS One 2016;11:e0164914.

18 Shah AM, Claggett B, Sweitzer NK, et al. Cardiac structure and function and prognosis in heart failure with preserved ejection fraction: findings from the echocardiographic study of the treatment of preserved cardiac function heart failure with an aldosterone antagonist (TOPCAT) trial. Circ Heart Fail 2014;7:740-51.

19 Nauta JF, Hummel YM, van der Meer P, et al. Correlation with invasive left ventricular filling pressures and prognostic relevance of the echocardiographic diastolic parameters used in the 2016 ESC heart failure guidelines and in the 2016 ASE/EACVI recommendations: a systematic review in patients with heart failure with preserved ejection fraction. Eur J Heart Fail 2018;20:1303-11.

20 Kang S-H, Park JJ, Choi D-J, et al. Prognostic value of NTproBNP in heart failure with preserved versus reduced EF. Heart 2015;101:1881-8.

21 Luchner A, Burnett JC, Jougasaki M, et al. Evaluation of brain natriuretic peptide as marker of left ventricular dysfunction and hypertrophy in the population. J Hypertens 2000;18:1121-8.

22 Kara K, Lehmann N, Neumann T, et al. Nt-proBNP is superior to BNP for predicting first cardiovascular events in the general population: the heinz Nixdorf recall study. Int J Cardiol 2015;183:155-61.

23 Portegies MLP, Kavousi M, Leening MJG et al N-terminal pro-Btype natriuretic peptide and the risk of stroke and transient ischaemic attack: the Rotterdam study. Eur J Neurol 2015;22:695-701.

24 Dietl A, Stark K, Zimmermann ME, et al. Nt-proBNP predicts cardiovascular death in the general population independent of left ventricular mass and function: insights from a large population-based study with long-term follow-up. PLoS One 2016;11:e0164060.

25 Redfield MM, Jacobsen SJ, Borlaug BA, et al. Age- and genderrelated ventricular-vascular stiffening: a community-based study. Circulation 2005;112:2254-62.

26 Gori M, Lam CSP, Gupta DK, et al. Sex-specific cardiovascular structure and function in heart failure with preserved ejection fraction. Eur J Heart Fail 2014;16:535-42.

27 Mottram PM, Haluska BA, Leano R, et al. Relation of arterial stiffness to diastolic dysfunction in hypertensive heart disease. Heart 2005;91:1551-6. 